\title{
Searching for a link between the magnetic nature and other observed properties of Herbig Ae/Be stars
}

\author{
Swetlana Hubrig ${ }^{1}$, C. Grady ${ }^{2}$, M. Schöller ${ }^{3}$, O. Schütz ${ }^{1}$, B. Stelzer ${ }^{4}$, \\ M. Pogodin ${ }^{5}$, M. Cure ${ }^{6}$ and R. Yudin 5 \\ ${ }^{1}$ ESO, Santiago, Chile; email: shubrig@eso.org \\ ${ }^{2}$ Eureka Scientific, Oakland, USA ${ }^{3}$ ESO, Garching, Germany \\ ${ }^{4}$ INAF-Osservatorio Astronomico di Palermo, Italy \\ ${ }^{5}$ Pulkovo Observatory, St.-Petersburg, Russia \\ ${ }^{6}$ Univ. de Valparaiso, Chile
}

\begin{abstract}
We present the results of a new magnetic field survey of Herbig Ae/Be and A debris disk stars. They are used to determine whether magnetic field properties in these stars are correlated with the mass-accretion rate, disk inclinations, companion(s), Silicates, PAHs, or show a more general correlation with age and X-ray emission as expected for the decay of a remnant dynamo.
\end{abstract}

Keywords. Stars: pre-main-sequence - stars: magnetic fields - X-rays: stars - techniques: polarimetric - stars: HD 101412, HD 139614, HD 144668, HD 152404, HD 190073

\section{Introduction}

Protoplanetary disks are where planets form, and where the pre-biotic materials, which produce life-bearing worlds, are assembled or produced. We need to understand them, how they interact with their central stars, and their evolution; both to reconstruct the Solar System's history, and to account for the observed diversity of exo-planetary systems. Our most detailed view of protoplanetary disks is for those surrounding intermediatemass stars, the Herbig Ae/Be stars (e.g. Herbig 1960) where the disks are revealed by their thermal emission, and in scattered light in the optical and near-IR.

\section{Observations and measurements}

The observations were carried out in May 2008 at the European Southern Observatory with FORS 1 mounted on the $8 \mathrm{~m}$ Kueyen telescope of the VLT. New magnetic field detections were achieved in eight stars. For three Herbig Ae/Be stars, we confirm the previous magnetic field detections. The star HD 101412, with the largest magnetic field strength measured in our sample stars using hydrogen lines, $\left\langle B_{\mathrm{z}}\right\rangle=-454 \pm 42 \mathrm{G}$, shows a change of the field strength by $\sim 100 \mathrm{G}$ during two consecutive nights. In Fig. 1 (left panel) we present distinct Zeeman features detected at the positions of the hydrogen Balmer lines and the $\mathrm{Ca}$ II H\&K lines. The $\mathrm{H} \beta$ line in the Stokes $I$ spectrum is contaminated by the presence of a variable emission in the line core and was not included in our measurements. Strong distinct Zeeman features at the positions of the Ca II H\&K lines are detected in four Herbig Ae/Be stars, HD 139614, HD 144668, HD 152404, and HD 190073. In Fig. 1 (right panel) we present the Stokes $V$ spectra for these stars in the region around the Ca II doublet, together with our previous observation of HD 190073. As we already reported 

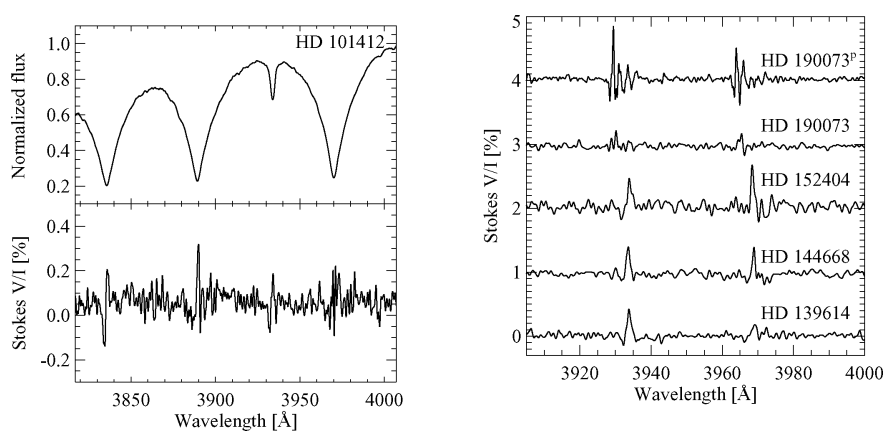

Figure 1. Left panel: Stokes $I$ and $V$ spectra in HD 101412; Right panels: Stokes $V$ spectra in the vicinity of the Ca II H\&K lines of the Herbig Ae/Be stars HD 139614, HD 144668, HD 152404, and HD 190073. At the top we present the previous observation of HD 190073, obtained in May 2005. The amplitude of the Zeeman features in the Ca II H\&K lines observed in our recent measurement has decreased by $\sim 0.5 \%$ compared to the previous observations.

in our earlier studies (Hubrig et al. 2004; Hubrig et al. 2006; Hubrig et al. 2007a) these lines are very likely formed at the base of the stellar wind, as well as in the accretion gaseous flow, and frequently display multi-component complex structures in both the Stokes $V$ and Stokes $I$ spectra.

\section{Looking for the links}

Most of our sample targets are Herbig Ae stars with masses of $3 \mathrm{M}_{\odot}$ and less. Since the observations of the disk properties of intermediate mass Herbig Ae stars suggest a close parallel to T Tauri stars, revealing the same size range of the disks, similar optical surface brightness and similar structure consisting of inner dark disk and a bright ring, it is quite possible that especially the magnetic fields play a crucial role in controlling accretion onto, and winds from, Herbig Ae stars, similar to the magnetospheric accretion observed in T Tauri stars. We do not find any trend between the presence of a magnetic field, disk inclination angles and mass-accretion rates. Also the membership in binary or multiple systems does not seem to have any impact on the presence of a magnetic field, whereas we find a hint that the appearance of magnetic fields is more frequent in Herbig stars with flared disks and hot, inner gas. The stronger magnetic fields tend to be found in younger Herbig stars. The magnetic fields become very weak or completely disappear in stars when they arrive on the ZAMS, clearly confirming the conclusions of Hubrig et al. (2000) and Hubrig et al. (2007b) that magnetic Ap stars with masses less than $3 \mathrm{M}_{\odot}$ are only rarely found close to the ZAMS. We also find a hint for an increase of the magnetic field strength with the level of the X-ray emission, suggesting a dynamo mechanism to be responsible for the coronal activity in Herbig Ae stars.

\section{References}

Herbig, G. H. 1960, ApJS 4, 337

Hubrig, S., North, P., \& Mathys, G. 2000, ApJ 539,352

Hubrig, S., Schöller, M., \& Yudin, R. V. 2004, A\&\&A 428, L1

Hubrig, S., Yudin, R. V., Schöller, M., \& Pogodin, M. A. 2006, A\&A 446, 1089

Hubrig, S., North, P., \& Schöller, M. 2007b, AN 328, 475

Hubrig, S., Pogodin, M. A., Yudin, R. V., et al. 2007a, A\&A 463, 1039 